\title{
REFLEXÕES SOBRE A RELAÇÃO SAÚDE E AMBIENTE: A PERCEPÇÃO DE UMA COMUNIDADE
}

\author{
M. A. BUSATO*, L. FERRAZ e N. L. P. FRANK \\ Universidade Comunitária da Região de Chapecó - UNOCHAPECÓ - Chapecó-SC \\ assunta@unochapeo.edu.br*
}

Artigo submetido em maio/2014 e aceito em novembro/2015

DOI: $10.15628 /$ holos.2015.2145

\section{RESUMO}

Cada indivíduo ou coletivo percebe e interage com o ambiente de forma diferente, influenciados pela sua formação, cultura, condição socioeconômica e capacidade cognitiva. Este estudo identificou a percepção de uma população sobre o meio ambiente e a relação com a saúde. A pesquisa foi realizada com 1023 famílias e os dados obtidos por meio de um formulário estruturado. A população referiu-se à presença de lixo nas ruas embora grande parte $(80,4 \%)$ garante realizar a separação. A presença de poluição da água, ar e do solo foi indicada por $42 \%$, além da presença de animais abandonados e, a maioria (52,3\%), identifica cheiro desagradável no ambiente. Os elementos que proporcionam a capacidade de percepção das condições do ambiente, explicitando situações que necessitam de melhorias representam riscos à sua saúde e podem ser entendidos como a capacidade de resposta que é um dos principais elementos da vulnerabilidade.

PALAVRAS-CHAVE: meio ambiente, saúde, comunidade, percepção.

\section{REFLECTIONS ON THE RELATIONSHIP HEALTH AND ENVIRONMENT: THE PERCEPTION OF A COMMUNITY}

\section{ABSTRACT}

Each individual or collective perceives and interacts with the environment differently, influenced by his training, culture, socioeconomic status, and cognitive ability. This study identified the perception of a population on the environment and the relationship to health. The survey was conducted with 1023 families and data obtained through a structured questionnaire. The population referred to the presence of garbage in the streets although most $(80.4 \%)$ ensures perform the separation.
\end{abstract}

The presence of water pollution, air and soil was indicated by $42 \%$, and the presence of abandoned animals, and the majority (52.3\%), identifies unpleasant smell in the environment. The elements that provide the ability to perceive environmental conditions, explaining situations that need improvement, they represent health risks can be understood as responsiveness which is a key element of vulnerability.

KEYWORDS: environment, health, community, perception. 


\section{INTRODUÇÃO}

A percepção de cada indivíduo sobre o ambiente em que vive bem como a forma que interage é diferente para cada um. As respostas ou manifestações são resultado das percepções (individuais e coletivas), dos processos cognitivos, julgamentos e expectativas de cada pessoa, sendo influenciadas também por elementos culturais (VASCO; ZAKRZEVSKI, 2010). Neste sentido, os conceitos formulados sobre saúde, ambiente, meio ambiente, saúde ambiental ou outros, podem ser percebidos de diferentes formas. Mesmo assim, alguns conceitos são pré-definidos.

Em 1948, a definição de saúde, proposta pela Organização Mundial da Saúde (OMS) afirma que afirma o dever do Estado em garanti-la, sendo direito de todos, e conceitua: "Saúde é o estado do mais completo bem-estar físico, mental e social e não apenas a ausência de enfermidade" (SCLIAR, 2007). Saúde Ambiental é entendida como a interação entre a saúde da população e os riscos para o desenvolvimento de agravos e doenças após exposição de certos elementos físicoquímicos (TAMBELLINI; CÂMARA, 1998). Para esses autores o conceito de ambiente é socialmente determinado, ampliando o caráter biológico, passando a compreender o ambiente como uma questão social. A inter-relação do ambiente e a saúde no processo saúde-doença do indivíduo deixam de ser uma dimensão externa ao homem, passando para uma condição de interdependência das demais dimensões da vida do ser humano. O ambiente contempla a relação que se estabelece entre o próprio ambiente e as condições de vida e saúde de uma população, que é dada pelas relações sociais e com o meio.

Para conceituar meio ambiente, Jacobi (1995) menciona que o espaço físico alterado pela atividade humana e o entendimento das questões ambientais estão relacionados à forma de atuação social e cultural na vivência deste meio. As alterações na paisagem são produzidas pela atuação dos agentes naturais e pelo homem. O documento sobre Vulnerabilidade Ambiental do Ministério do Meio Ambiente refere que a maneira como o ambiente se encontra é resultado das ações passadas e como está em constantes modificações será diferente no futuro (BRASIL, 2007).

A relação entre ambiente e saúde está sendo pauta de fóruns nacionais e internacionais nos últimos anos, a exemplo da I Conferência Nacional de Saúde Ambiental, no Brasil, em 2009, cuja discussão foi feita em todos os municípios e estados e os encaminhamentos foram, dentre outros, a atenção para a conservação do meio ambiente e a minimização dos riscos à saúde humana decorrentes dos agravos ambientais, pois não há como dissociar questões relacionadas à saúde do contexto ambiental. Em 2009, nas conferências municipais, estaduais e nacional, todo o Brasil discutiu de forma participativa, as questões relativas à Saúde Ambiental. Nesses encontros, as temáticas de educação ambiental, saúde ambiental, saúde humana foram debatidas de modo a (re)pensar e elaborar caminhos para a melhoria do ambiente físico-social.

No que diz respeito à educação ambiental, na visão de Letff (2002), o seu processo deve ocorrer visando à integração inter e transdisciplinar do conhecimento, no diálogo entre os saberes e na articulação entre as ciências; não na combinação, pura e simplesmente, de todos os conhecimentos já consolidados, mas sim, na adição das diferenças e diversidades. Deste modo torna-se imprescindível uma prática educativa que considere e valorize o conhecimento prévio e a cultura numa perspectiva Freireana, destacando que "só existe saber na invenção, na reinvenção, na busca inquieta, impaciente, permanente, que os homens fazem do mundo, com o mundo e com 
os outros" (FREIRE, 2004, p. 58). Nesse sentido, a realização de estudos sobre a percepção ambiental é uma importante ferramenta política, pois revela e contextualiza a realidade local e possibilita subsídios para o planejamento e a gestão, evitando ou minimizando os conflitos entre o homem e o ambiente (GUEDES et al., 2013).

Considerando as diferentes formas de perceber e dar respostas, este estudo teve como objetivo identificar a percepção de uma população acerca do meio ambiente em que vive e reflexionar sobre sua relação com a saúde e ambiente.

\section{PROCEDIMENTOS METODOLÓGICOS}

A pesquisa realizada compreendeu um estudo transversal de natureza exploratóriodescritiva no território de um dos Núcleos de Apoio à Saúde da Família (NASF) do município de Chapecó - SC. O território foi selecionado pela Universidade em parceria com a Secretaria da Saúde do município partícipe no Programa Nacional de Reorientação da Formação Profissional em Saúde (Pró-Saúde).

Compuseram a amostra 1023 famílias cadastradas nos centros de saúde inseridos que formam esse NASF, de um total de 13.866 famílias, cujos dados foram obtidos do Sistema de Informação de Atenção Básica (SIAB). Para estabelecer o tamanho dessa amostra foi considerado um erro amostral de $3 \%$ e uma proporção de perda em $10 \%$.

Os domicílios foram selecionados aleatoriamente por meio das fichas dos cadastros dos agentes comunitários de saúde (ACS) pertencentes aos centros de saúde do estudo. Num primeiro momento foram reunidos os prontuários das famílias de acordo com sua microárea de residência. Posteriormente foi selecionada a oitava família (número sorteado pela equipe de pesquisadores) e, a partir dessa, as próximas subsequentes com intervalo de 12 prontuários, até atingir o número da amostra determinado para cada centro de saúde. Na terceira etapa, houve a confirmação com o ACS responsável pela família, se esta ainda residia no endereço registrado e se havia restrição de horário para a visita. No caso de impossibilidade de contato, fez-se sorteio de uma nova família.

A coleta de dados foi obtida através da aplicação de um formulário com questões estruturadas abrangendo temas relativos às condições de vida da população e ao ambiente comunitário. Para a formulação do instrumento, além da equipe de pesquisadores, houve a participação de profissionais da saúde da Atenção Básica, com o intuito de qualificar as informações colhidas.

A pesquisa foi desenvolvida com a atuação de 108 acadêmicos, 48 profissionais da saúde (preceptores da Secretaria da Saúde de Chapecó) e 14 docentes dos programas Programa Nacional de Reorientação da Formação Profissional em Saúde (Pró-Saúde), Programa de Educação pelo Trabalho para a Saúde (Pet-Saúde) e Programa de Educação pelo Trabalho - Vigilância em Saúde (Pet-Visa), 12 tutores da Universidade Comunitária da Região de Chapecó e dois coordenadores dos programas. A coleta dos dados nos domicílios foi executada basicamente pelos acadêmicos orientados pelos profissionais da saúde. A avaliação e manejo dos dados foram realizadas pelos docentes dos programas sob a supervisão das coordenadoras da pesquisa.

Para o tratamento dos dados foram aplicadas técnicas próprias de um estudo descritivo, as quais informam sobre as médias e a frequência (absoluta e relativa) da distribuição de um determinado evento na população em estudo. Os dados coletados foram codificados, tabulados e 
digitados em bancos de dados, especialmente construídos no programa Statistical Package for Social Science (SPSS). Após a digitação, os dados sofreram o processo de controle de qualidade com análise de coerência e consistência.

Com relação aos aspectos éticos da pesquisa, o projeto foi aprovado pelo Comitê de Ética em Pesquisa com Seres Humanos (CEP) da Unochapecó, sob Parecer no 001/2011. Quanto aos participantes do estudo, foi explicado o propósito, os objetivos, procedimentos da pesquisa e solicitada a assinatura de um termo de consentimento livre e esclarecido. Também foi garantido aos participantes o direito de retirarem-se da pesquisa, caso assim o desejassem, bem como a proteção da identidade, o respeito à individualidade e à privacidade de todos os envolvidos. Os protocolos usados na pesquisa estão de acordo com a Resolução 466/2012 do Conselho Nacional de Saúde/Ministério da Saúde.

\section{O AMBIENTE NO OLHAR DA COMUNIDADE}

No estudo realizado com as 1023 famílias foi possível identificar a percepção da comunidade com relação ao ambiente em que vive. A caracterização do perfil sociodemográfico dessas famílias é: a maioria dos respondentes das entrevistas é do sexo feminino, com idade média de 46,3 anos. As famílias são compostas, em média, por 3,5 pessoas. Dessas, $6,4 \%$ recebem algum tipo de auxílio do governo federal, como Bolsa Família e Auxílio Alimentação do Serviço de Assistência Social da prefeitura de Chapecó. Em 17,8\% das famílias há, pelo menos, um membro que busca trabalho/emprego. A renda média familiar é de 2,22 salários mínimos, porém a renda per capita é de 0,63 salários mínimos. 27,8\% têm, pelo menos, um membro que não sabe ler ou escrever. Em 15,7\% há um familiar com ensino superior completo, 9,9\% com pós-graduação e em 14,4\% há, pelo menos, um estudante cursando o ensino superior. Em relação às condições de moradia, 59,3\% dos entrevistados relatam que consideram suas casas em boas condições.

Alguns dos itens identificados neste estudo foi a percepção sobre a presença de poluição da água, ar e do solo. Para $42 \%$ dos entrevistados há poluição em todos esses ambientes, o que evidencia, à primeira vista, que são necessárias ações de melhoria e cuidados com o ambiente em que vive essa população cujas condições podem interferir na sua qualidade de vida e saúde. Uma situação de qualidade do ambiente com implicação na saúde foi registrada por Gouveia et al. (2006) que identificaram associação entre altos índices de poluentes atmosféricos e o número de internações de crianças e idosos na cidade de São Paulo. Sobre este aspecto avaliaram a associação entre a poluição do ar e internações de crianças e idosos em hospitais da rede pública de saúde. Para os autores os efeitos provocados pela poluição do ar podem surgir dias após a exposição e afetar a saúde da população. Da mesma forma, a poluição da água tem efeito sobre a saúde sob diferentes aspectos, sejam doenças relacionadas às infecções gastrointestinais ou favorecimento ao desenvolvimento de vetores transmissores de doenças.

Na percepção dos moradores de uma favela do sul do Brasil acerca do ambiente (LERMEN; FISHER, 2010), a população aponta que tem sua saúde afetada pela poluição local devido a produtos químicos, lixo, ruído e barulho. Se, por um lado, os autores indicam que a consciência ambiental constituiu-se como meio primordial de resolução dos problemas do meio ambiente, por outro, a população acredita que a responsabilidade seja basicamente do governo municipal e da empresa de coleta de lixo, excluindo a participação da comunidade na questão. No entanto, Silva et al. (2012) reiteram que a falta de informação leva à incompreensão de problemas, o que 
aumenta as chances dos moradores estagnarem na condição sócio-ambiental, reduzindo as chances de melhorias na qualidade de vida.

A população deste estudo referiu-se à presença de lixo e entulho nas ruas e ambientes públicos, embora grande parte $(80,4 \%)$ garante realizar constantemente a separação de lixo em suas residências. Nesta constatação identifica-se que há preocupação no cuidado com os resíduos quando a população se refere à separação do lixo, já que este é o primeiro passo para o cuidado com o local do ambiente em que vive. No entanto, a porta de saída dos resíduos é de responsabilidade individual e do coletivo público, havendo a necessidade do destino correto do material, contudo somente $42 \%$ da população percebe esse fator - presença de lixo inadequadamente - como um problema de saúde ambiental.

Quando ocorre o correto destino dos resíduos, sem o aspecto visual de "lixo na rua", podese considerar a melhora da saúde da população e do ambiente assim como foi percebido por Monteiro e Nazário (2000) nas décadas de 1980 e 1990, em São Paulo, quando compararam dados de bairros residenciais e favelas avaliando a associação entre saúde, condições de vida e ambiente. Os autores observaram que a intervenção do poder público devido à preocupação com indicadores de saúde e a qualidade de vida da população, foram feitas melhorias significativas, especialmente, nos aspectos da coleta de lixo e aumento da rede de esgoto. Neste contexto, Bay e Silva (2011) reiteram que o grau de evolução de uma comunidade é identificado pela forma como ela trata seus recursos hídricos e seu lixo e, ratificam que, da mesma forma, identifica-se a seriedade e competência de uma administração pelos esforços em prol do saneamento.

Um aspecto de percepção de ambiente que seja bom de viver é, além do visual, o do odor. Neste estudo, a maioria (52,3\%) dos entrevistados percebe cheiro desagradável no ambiente. Quando se remete ao tema cheiro desagradável, é imprescindível relembrar o que diz a obra de Alain Corbin, escrita em 1970, na qual o historiador francês descreve a "revolução olfativa" associada à maior frequência no uso de novas técnicas higiênicas. O livro tem como base a ideia de que, por meio da intolerância aos maus odores, houve uma mudança comportamental ocorrida entre os séculos XVIII e XIX (SILVA, 2012). Corbin relata que anteriormente à revolução olfativa, em Madri, os dejetos fecais eram espalhados pelas ruas e, em Londres, as fossas sépticas ficavam abertas para espantar e combater a peste, servindo de medida protetora para a saúde da população. Na concepção da época, os maus odores poderiam causar certo desconforto, mas não eram considerados insalubres. Porém, gradativamente, com os novos pensamentos sobre higienização, também ocorreram mudanças na sensibilização aos odores, tornando-os insuportáveis (SILVA, 2012).

Um ambiente sem odores desagradáveis, de aspecto visual limpo, sem lixo e resíduos de esgoto a céu aberto é um condicionante de qualidade de saúde e ambiente, sendo preventivo à presença de doenças. É nessa direção que Silva (2012) destaca que o processo de higienização e alteração da sensibilização aos odores resulta em medidas de modernização e resolução de alguns problemas sociais, além da transformação no comportamento das pessoas, destacando-se a melhoria na qualidade de vida.

Além do cheiro desagradável, outro problema social e de saúde na comunidade estudada é a presença de animais abandonados nas ruas, praças e, inclusive, adentrando nos pátios das casas. A maioria da população $(74,3 \%)$ relata a presença de animais soltos nas ruas da comunidade sendo que $43,30 \%$ mencionaram que há muitos animais soltos. Os animais de rua necessitam 
alimentar-se de outros animais menores ou mesmo lixo doméstico, propiciando a exposição a vários vetores de doenças, o que facilita a propagação das zoonoses (VIANNA, 2003). A relação de proximidade entre o homem e animais de rua, seja pelo contato direto ou pela coabitação nos espaços públicos, pode resultar em danos à saúde se não houver um controle rigoroso de combate às zoonoses, pois os dejetos eliminados por animais parasitados podem causar doenças na população (CORRÊA; GRUNSPAN; LAGAGGIO, 1993).

Os animais perambulantes, especialmente gatos e cachorros, podem ser veiculadores de zoonoses. Uma forma de erradicar as zoonoses é conter a população de animais nas ruas, pois estes ficam mais expostos à contaminação pelos micro-organismos causadores das doenças, servindo de reservatório e auxiliando na disseminação das zoonoses cujas infecções podem se desenvolver tanto em animais como no ser humano. Em virtude do risco à saúde das pessoas e animais, precauções para a prevenção devem ser tomadas a fim de combater possíveis doenças. Milano e Oscherov (2002) recomendam a necessidade de que a população, que se encontra em condições de risco por possíveis agressões pelos animais soltos nas ruas, tenha conhecimento sobre essas doenças. Para minimizar essa vulnerabilidade, é importante a atuação conjunta dos setores da saúde e da gestão ambiental para levar informações acerca da transmissão e prevenção das doenças.

Além dos animais de rua, a presença de vetores transmissores de enfermidades também é apontada pela população como uma situação de risco para a presença de dengue, por exemplo, tendo em vista a notificação de focos do vetor Aedes aegypti tanto na região de estudo como em todo o município.

A presença de vetores transmissores de enfermidades pode ocorrer devido ao descuido com o lugar quando não há destinação correta dos resíduos e o saneamento é deficitário. Para Fonseca (2012), a limpeza e o cuidado se configuram como formas de proteção da saúde em relação ao meio, sendo medidas de proteção da saúde, de forma que o não cumprimento acarretaria em doença e mal estar. No entanto, em não havendo esse equilíbrio, Waltner-Toews (2001) afirma que ocorre uma expansão ou possibilidade de novos habitats para fauna e flora que causam doenças, quando são realizados programas que se propõem a promover saúde em algumas dimensões como, por exemplo, por meio da reestruturação ambiental para melhoria do suprimento de alimento, energia e água e removendo fontes de renovação natural da terra. A percepção sobre o ambiente que uma população tem sobre seu espaço representa a paisagem daquele lugar, no entanto, na concepção de Collot (1990), não se pode falar de paisagem a não ser a partir de sua percepção, pois ela se define como um espaço percebido.

\section{O AMBIENTE E SAÚDE: UMA REFLEXÃO}

O ambiente é tudo o que cerca o indivíduo, aí compreendida a interação entre elementos naturais e sociais, é construído, moldado e ganha significado por meio da apropriação humana (FAHEL; CAMPOS; ARAÚJO, 2006). Nessa perspectiva, pondera-se que os aspectos biológico, social, psicológico e ambiental estão diretamente ligados às condições de saúde de uma população. As condições do ambiente fazem parte do conceito ampliação de saúde incorporando o saneamento, a água para consumo humano, a poluição química, a pobreza, bem como a percepção que cada sujeito tem do seu lócus. 
De acordo com a VIII Conferência Nacional de Saúde (CNS), "a saúde é a resultante das condições de alimentação, habitação, educação, renda, meio ambiente, trabalho, transporte, emprego, lazer, liberdade, acesso e posse da terra e acesso aos serviços de saúde" (BRASIL, 1986 p. 4).

A concepção de saúde é compreendida não como a ausência de doença, mas o estado do produto social resultante de fatores políticos, econômicos, ideológicos, ambientais e culturais. Nessa perspectiva, o processo saúde-doença está relacionado à forma pela qual as pessoas interagem com os eventos do cotidiano. Reconhecendo que o processo saúde-doença está relacionado a fenômenos complexos para a análise sobre o processo saúde-doença de uma população, em um dado território, é necessário identificar a relação entre as distintas variáveis, entre as quais os fatores socioambientais adquirem especial relevância. Esses fatores incluem os biológicos, psicológicos, sociais, culturais, econômicos e ambientais.

Nos diagnósticos de condições de vida e de situação de saúde, os elementos constitutivos da reprodução da vida social nos diversos lugares não podem ser tratados como conteúdos desarticulados do ambiente/território. A consideração dos aspectos ambientais como estratégia de análise sobre condições de saúde e intervenção pressupõe a identificação de objetos geográficos, sua utilização pela população e sua importância para os fluxos das pessoas e materiais. Para isso, é necessário o desenvolvimento de metodologias para o reconhecimento, em campo ou mediante dados secundários, de objetos e suas formas, que são condições da ação e meios de existência do agir humano (MONKEN; BARCELLOS, 2005).

Segundo Freitas (2003), problemas ambientais são, simultaneamente, problemas de saúde, pois a preocupação das condições ambientais sobre a saúde é evidente desde a antiguidade, em que os efeitos do clima interferiam no balanço dos humores do corpo, nos miasmas, nas sujeiras e nos odores. E, "assim, sempre esteve presente nos diferentes discursos e práticas sanitárias que se constituíram como respostas sociais às necessidades e aos problemas de saúde" (p. 138).

Buss (2000) enfatiza que para construir ambientes salubres alude considerar a complexidade das relações sociais e de interdependência entre diversos setores da sociedade, partindo da proteção do meio ambiente ao impacto que das mudanças ambientais produzem sobre o processo saúde-doença.

Nessa perspectiva, Camponogara et al. (2013) defendem a importância da valorização da inter-relação entre o meio ambiente e a promoção da saúde. Desse modo, os autores defendem a necessidade de ampliar o debate sobre a interface saúde e meio ambiente, pois essa é uma demanda contemporânea que exige do setor saúde à responsabilidade com bases teóricas e práticas compatíveis com pressupostos éticos, para esta e para as futuras gerações, na preservação do planeta.

Freitas et al. (2007), identificam duas vertentes básicas para uma abordagem ecossistêmica na saúde, a saber: a primeira é a mensuração de sinais e sintomas presentes no ecossistema que afetam a saúde, e está fundamentada em informações científicas. A outra é a resoluções dos problemas dos ecossistemas de contextos específicos, bem como as consequências das transformações deste ecossistema para a comunidade local. Nesse sentido, com o intenso e acelerado processo de desenvolvimento econômico e populacional pelo qual o mundo vem passando, Bruzos e colaboradores destacam que: 
É fundamental discutir a temática ambiental entre os profissionais da saúde, para que eles se empoderem desse conhecimento e consigam identificar problemas relacionados à questão ambiental, propondo ações resolutivas e preventivas, juntamente com a comunidade, procurando amenizar os riscos ambientais a que todos estão expostos. Reforça-se a profundidade do papel dos profissionais de saúde diante dos problemas ambientais, buscando a saúde em uma perspectiva ampliada de promoção da saúde (BRUZOS et al., 2011, p. 462).

A necessidade urgente de ampliar o debate sobre a interface saúde e meio ambiente, junto aos profissionais de saúde, é defendida por Camponogara et al. (2013), haja vista que exige do setor saúde o estabelecimento de bases teóricas e práticas compatíveis com os pressupostos éticos para a preservação da saúde do planeta.

A relação entre ambiente e saúde deve ser uma prática presente nas discussões de uma população coordenada, principalmente, pelos profissionais da saúde, já que são eles que lidam diretamente com essa realidade e a relação da conservação ambiental com as condições de saúde. Os profissionais da área da saúde podem construir junto com a população, mapeamento da realidade local e, a partir da educação ambiental, direcionar as ações para melhoria do ambiente.

O ambiente precisa ser agradável aos olhos de quem ali vive. Isso implica em um bem viver, pois ambiente e saúde são interdependentes e inseparáveis e fazem parte do desenvolvimento humano, implicando na melhoria da qualidade de vida. Na visão de Fonseca (2012), a saúde de uma população depende intimamente da complexa relação que esta estabelece com seu meio. 0 binômio saúde-doença se constitui como um processo coletivo sendo necessário, portanto (MINAYO, 2009), recuperar nesse coletivo o sentido de "lugar" como o espaço organizado para análise e intervenção, buscando identificar as relações entre condições de saúde e seus determinantes culturais, sociais e ambientais.

A qualidade do ambiente é de responsabilidade de seus habitantes adicionada à responsabilidade dos cuidados devidos ao poder público. No entanto, a percepção do ambiente expressa pela população estudada, tanto a visual como a sensorial, mostra que há necessidade de melhorias no território onde vivem. Ao que compete aos moradores, acredita-se que, nem sempre se apropriam do meio, cuidam como seu habitat ou têm a sensação de pertencimento, fatores esses imprescindíveis para a efetiva (co)participação no processo de melhoria do ambiente. É nessa tônica que Herculano, Porto e Freitas (2000) mencionam que quando os indivíduos refletem sobre si mesmos e suas ações sobre o ambiente, percebem que o transformam em sua própria imagem, pois o constroem e reconstroem, destroem e reformam, ou seja, o moldam a partir da cultura da sociedade em que vivem.

Nesta perspectiva, entende-se que as pessoas podem ter boa escolaridade, longa expectativa de vida, acesso às riquezas geradas, mas morarem e trabalharem em locais poluídos, sujeitos a riscos, conviverem com águas sujas, respirarem poluentes e habitarem compactamente selvas de pedra deprimentes, onde as cores predominantes sejam os tons de cinza do cimento e do asfalto, logo, não evidenciando a condição de saúde. Por isso, bem-estar tem de envolver também aspectos ambientais. Considera-se que os aspectos ambientais, onde está inserido tudo o que comporta o "meio ambiente", é a relação que se estabelece entre o próprio sujeito e o ambiente. 
A percepção da população sobre o ambiente, explicitando situações que necessitam de melhorias, evidencia que ela é capaz de detectar essas condições a partir de sua formação, cultura, condição socioeconômica e capacidade cognitiva. Embora haja conhecimento sobre os problemas percebidos, Camponogara, Ramos e Kirchhof (2009) expressam que parece não haver o esperado comportamento de preservação ambiental por parte de significativa parcela da população, resultando em dificuldades para efetivar ações. Os elementos que proporcionam a capacidade de percepção das condições do ambiente, os quais representam riscos à sua saúde, podem ser entendidos como a capacidade de resposta que é um dos principais elementos da vulnerabilidade. O reconhecimento dos problemas e o enfrentamento desses riscos mostram que a vulnerabilidade, como destaca Cutter (2003), envolve uma gama de fenômenos de natureza multidimensional e multifacetada que torna imperativo o diálogo e um olhar abrangente sobre o tema. Isso pode ser implementado tendo como origem ações da própria comunidade a partir do sentido de pertencimento do ambiente como "seu", ou do poder público por meio de políticas e ações que promovam debate sobre os pressupostos de "saúde e ambiente".

Para Minayo (2009) o primeiro desses pressupostos é a essencialidade da relação entre os seres humanos e a natureza e, o segundo, derivado dessa relação, é de que o conceito de ambiente é construído pela ação humana. Frente a isso, a relação existente entre saúde e ambiente, em todo o contexto em que essa relação demanda, deve ser premissa de atenção, em especial, na construção e desenvolvimento de políticas públicas. É necessário, portanto, que sejam observadas as especificidades locais uma vez que as cidades são heterogêneas, e, portanto, respondem aos desafios ambientais e sociais de formas diferenciadas (ARAUJO; CÂNDIDO, 2014).

A educação em saúde e a ambiental estão paralelamente tangidas, indicando a necessidade de não distanciá-las e tampouco trabalhar de modo isolado nos espaços comunitários. Nesse sentindo, deve-se considerar que tanto na educação em saúde como a ambiental é necessária uma pedagogia que valorize a educação dentro do contexto social, ecológico e cultural onde se situam os sujeitos (LEFF, 2002). Isso exige um olhar crítico, buscando compreender os problemas socioambientais em suas múltiplas relações, considerando o ambiente como o conjunto natural do mundo social, mediado por saberes locais, tradicionais e científicos (SILVA, 2007).

\section{CONSIDERAÇÕES FINAIS}

Da análise das percepções da população participante do estudo, nota-se que as famílias que fizeram parte deste estudo percebem a presença de poluição ambiental na comunidade onde vivem. Essa evidência se apresenta na medida em que reconhecem a presença de lixo nas ruas, animais soltos e o cheiro desagradável como fatores de degradação ambiental e de poluição.

No entanto, é importante ressaltar que as percepções sobre o ambiente em que as pessoas vivem estão relacionadas com os aspectos cognitivos, históricos e culturais de cada individuo. Deste modo, (re)conhecer a percepção dos indivíduos a respeito dos espaços em que vivem é primordial para o desenvolvimento de uma educação ambiental que, segundo Reigota (1994), não seja necessariamente uma prática pedagógica voltada para a transmissão de conhecimentos sobre ecologia, nem vise somente à utilização racional dos recursos naturais, mas que contemple à participação dos cidadãos, com suas percepções, nas discussões e decisões sobre a questão do meio ambiente. 
Entende-se que cada indivíduo pode responder, agir e reagir de forma diferente às interferências e relações com o meio em que vive. No entanto, o comportamento dado como respostas, bem como suas manifestações decorrentes de sua percepção, normalmente são dadas de forma coletiva ou individual, levando em consideração a formação do sujeito, sua capacidade cognitiva e sua cultura.

Além disso, as políticas públicas de promoção de ambientes saudáveis precisam considerar a sociedade civil como corresponsável e proativa na sustentabilidade ambiental do território em que as pessoas vivem e extraem os recursos para a sua existência. Nesse sentido, a educação ambiental, além de ocorrer nas escolas para o desenvolvimento da consciência ecológica nas crianças, deve ser realizada nos espaços de atuação do setor saúde, para que população compreenda e valorize a inter-relação entre ambiente, saúde e bem-estar. Igualmente, sugere-se que os profissionais de saúde tenham como estratégia de promoção ao autocuidado, a sensibilização da população sobre os aspectos ambientais, incorporando nas suas ações assistências as dimensões do homem-natureza, evidenciando os riscos e exposições, os meios de proteção e de enfrentamentos dos problemas ambientais que afetam a saúde.

\section{REFERÊNCIAS}

1. ARAUJO, M.C.C.; CÂNDIDO, G.A. Qualidade de vida e sustentabilidade urbana. HOLOS, ano 30, v.1, p.3-19, jan./fev. 2014.

2. BAY, A.M.; SILVA, V.P. Percepção ambiental de moradores do bairro de liberdade de Parnamirim/RN sobre esgotamento sanitário. HOLOS, n.27, v.3, p.97-112, mai./jun. 2011.

3. BRASIL. Ministério do Meio Ambiente. Vulnerabilidade ambiental. Brasília: Ministério do Meio Ambiente, 2007.

4. BRASIL. VIII Conferência Nacional de Saúde. Relatório final. Brasília, 1986.

5. BRUZOS, G.A.S.; KAMIMURA, H.M.; ROCHA, S.A.; JORGETTO, T.A.C.; PATRÍCIO, K.P. Meio ambiente e enfermagem: suas interfaces e inserção no ensino de graduação. Saúde e Sociedade, v.20, n.2, p.462-469, abr./jun. 2011.

6. BUSS, P.M. Promoção da saúde e qualidade de vida. Ciência \& Saúde Coletiva, v.5, n.1, p.163177, jan./jul. 2000.

7. CAMPONOGARA, S.; RAMOS, F.R.S.; KIRCHHOF, A.L.C. Reflexividade, conhecimento e consciência ecológica: premissas para uma ação responsável no contexto do trabalho hospitalar. Revista Latino-americana de Enfermagem, v.17, n.6, p.1-8, nov./dez. 2009.

8. CAMPONOGARA, S.; VIERO, C.M.; ERTHAL, G.; SILVA DIAZ, P.S.; ROSSATO, G.C.; SOAES, S.A.; PERES, R.R. Visão de profissionais e estudantes da área de saúde sobre a interface saúde e meio ambiente. Trabalho, Educação e Saúde, v.11, n.1, p.93-111, jan./abr. 2013.

9. COLLOT, M. Pontos de vista sobre a percepção das paisagens. Boletim de geografia teorética, v.20, n.39, p.21-31, jan. 1990.

10. CORRÊA, G.L.B.; GRUNSPAN, E.; LAGAGGIO, V.R. Pesquisa de ovos e oocistos em fezes de cães e gatos, em praças públicas de Santa Maria e sua importância na clínica veterinária e em saúde pública. In: Anais do Congresso Internacional de Medicina Veterinária em Língua Portuguesa, Salvador, 1993. 
11. CUTTER, S.L.; BORUFF, B.J.; SHIRLEY, W. L. Social Vulnerability to Environmental Hazards. Social Science Quarterly, v.84, n.2, p.242-261, jun. 2003.

12. FAHEL, M.; CAMPOS, M.; ARAÚJO, C. Configuração (dilemas) dos riscos ambientais e de saúde: tendências e perspectivas no Brasil. InterfacEHS, v.1, n.2, artigo 6, dez. 2006.

13. FONSECA, A.F.Q. Ambiente e saúde: visão de profissionais da saúde da família. Revista Ambiente \& Sociedade, v. XV, n.2, p.133-50, maio./ago. 2012.

14. FREITAS, C.M. Problemas ambientais, saúde coletiva e ciências sociais. Ciência \& Saúde Coletiva, v.8, n.1, p.137-150, jan./mar. 2003.

15. FREITAS, C.M.; OLIVEIRA, S.G.; SCHÜTZ, G.E.; FREITAS, M.B.; CAMPONOVO, M.P.G. Ecosystem approaches and health in Latin America. Cadernos de Saúde Pública, v.23, n.2, p.283-296, fev. 2007.

16. FREIRE, P. Pedagogia da Autonomia: saberes necessários à prática educativa. 29 ed. São Paulo: Paz e Terra, 2004.

17. GOUVEIA, N.; FREITAS, C.U.; MARTINS, L.C.; MARCILIO, I.O. Hospitalizações por causas respiratórias e cardiovasculares associadas à contaminação atmosférica no Município de São Paulo, Brasil. Cadernos de Saúde Pública, v.22, n.12, p.2669-2677, dez. 2006.

18. GUEDES, F.A.F.; FRANCO, M.W.; MAIA-BARBOSA, P.; DRUMOND, M.A.; BARBOSA, F.A.R. Percepção ambiental dos moradores de São José do Goiabal sobre o parque estadual do Rio Doce: a influência das variáveis gênero, idade, classe social e escolaridade. Pesquisa em Educação Ambiental, v.8, n.1, p.51-61, jan./jul. 2013.

19. HERCULANO, S.; PORTO, M.F.S.; FREITAS, C.M. (Org.). Introdução: qualidade de vida e riscos ambientais como campo interdisciplinar em construção. In: Qualidade de Vida e Riscos Ambientais. Niterói: EdUFF, 2000. 334p.

20. JACOBI, P. Environmental problems facing urban households in the city of São Paulo, Brazil. Stockholm: Stockholm Environment Institute, 1995.

21. LEFF, E. Epistemologia Ambiental. 2 ed. São Paulo: Cortez, 2002.

22. LERMEN, H.S.; FISHER, P.D. Percepção ambiental como fator de saúde Pública em área de vulnerabilidade social no Brasil. Revista APS, v.13, n.1, p.62-71, jan./mar. 2010.

23. MILANO, A.M.F.; OSCHEROV, E.B. Contaminación por parasitos caninos de importancia zoonotica en playas de la ciudad de Corrientes, Argentina. Parasitología Latinoamericana, v.57, n.3-4, p.119-123, jul. 2002.

24. MINAYO, M.C.S. Saúde e ambiente: uma relação necessária. In: MINAYO et al. (orgs.). Tratado de Saúde Coletiva. 2ª ed. Rio de Janeiro: FIOCRUZ, 2009.

25. MONKEN, M.; BARCELLOS, C. Vigilância em saúde e território utilizado: perspectivas teóricas. Cadernos de Saúde Pública, v.21, n.3, p. 898-906, maio./jun. 2005.

26. MONTEIRO, C.A.; NAZARIO, C.L. Evolução de condicionantes ambientais da saúde na infância na cidade de São Paulo (1984-1996). Revista de Saúde Pública, v.34, n.6, p.13-18, nov./dez. 2000.

27. REIGOTA, M. Meio ambiente e representação social. São Paulo: Cortez, 1994.

28. SCLIAR, M. História do conceito de saúde. Physis. Revista de Saúde Coletiva, v.17, n.1, p. 29- 
41, jan./abr. 2007.

29. SILVA, C.M. A história cultural: um diálogo entre Alain Corbin e Norbert Elias. Fênix - Revista de História e Estudos Culturais, v. 9, n. 1, jan/abr. 2012.

30. SILVA, C.A.F.; MALAFAIA, G.; VEIGA, B.G.A. CASTRO, A.L.A. Vulnerabilidade ambiental e social: estudo de caso em um bairro no município de Sales-SP. HOLOS, v.4, Ano 28, p.45-54, jul./ago. 2012.

31. SILVA, A.L. Da ecologia social à educação ambiental: as contribuições do pensamento libertário de Murray Bookchin. Dissertação 176f. (Mestrado em Educação Ambiental). Universidade Federal do Rio Grande. Rio Grande, 2007.

32. TAMBELLINI, A.T.; CÂMARA, V.M. A temática saúde e ambiente no processo de desenvolvimento do campo da saúde coletiva: aspectos históricos, conceituais e metodológicos. Ciência \& Saúde Coletiva, v.3, n.2, p.47-59, ago./dez. 1998.

33. VASCO, A.P.; ZAKRZEVSKI, S.B.B. O estado da arte das pesquisas sobre percepção ambiental no Brasil. Perspectiva, v.34, n.125, p.17-28, mar. 2010.

34. VIANNA, M.S.R. Legislação de apoio ao controle de zoonoses. Rio de Janeiro: Secretaria Municipal de Saúde, 2003.

35. WALTNER-TOEWS, D. An ecosystem approach to health and its implications to

36. tropical and emerging diseases. Cadernos de Saúde Pública, v 17, (Suplemento), p.7-36, 2001. 